\title{
A comunicação alternativa como suporte a indivíduos com esclerose lateral
}

\section{amiotrófica}

\author{
Alternative communication in support of individuals amyotrophic lateral sclerosis \\ Comunicación alternativa en apoyo de personas con esclerosis lateral amiotrófica
}

Francisca Laudênia Vieira Ribeiro

ORCID: https://orcid.org/0000-0003-4155-2146

Faculdade de Ensino Superior do Piauí, Brasil

E-mail: laude-nia@hotmail.com

Gislene Mariana Pereira Castelo Branco

ORCID: https://orcid.org/0000-0003-4393-771X Faculdade de Ensino Superior do Piauí, Brasil

E-mail: gi.marianna@hotmail.com

Maria Lucilene da Silva

ORCID: https://orcid.org/0000-0003-0575-6096 Faculdade de Ensino Superior do Piauí, Brasil

E-mail: lucilene27@gmail.com

Tamires de Moura Saboia

ORCID: https://orcid.org/0000-0003-3060-7834

Faculdade de Ensino Superior do Piauí, Brasil

E-mail: fonotamsaboia@gmail.com

Ruth Raquel Soares de Farias

ORCID: https://orcid.org/0000-0002-0988-0900

Faculdade de Ensino Superior do Piauí, Brasil E-mail: ruthraquelsf@gmail.com

\begin{abstract}
Resumo
A Comunicação Alternativa é um sistema de comunicação que proporciona ao indivíduo, em diferentes fases da vida, a possibilidade de se comunicar em diversos contextos sociais. Assim, vários recursos são utilizados para contribuir com o indivíduo com Esclerose Lateral Amiotrófica - ELA, a qual é uma doença caracterizada por deterioração de neurônios motores causa paralisia progressiva de músculos esqueléticos, comprometendo a motricidade dos membros, da deglutição, da respiração e da fala. O comprometimento da fala tem papel fundamental na qualidade de vida do paciente bem como na de seu cuidador. A Comunicação Alternativa Ampliada (CAA) surge como uma oportunidade para amenizar as limitações de comunicação impostas pela doença devido às dificuldades apresentadas pela doença são necessários meios que facilitem a continuidade das atividades cotidianas. O presente trabalho objetiva analisar alternativas, para a expressão do pensamento, a uma pessoa impedida, devido à doença, de estabelecer efetiva comunicação verbal oralizada, escrita ou sinalizada. A metodologia da pesquisa consiste em uma uma pesquisa bibliográfica do tipo integrativa, por meio da análise de teorias, presentes em várias fontes, como por exemplo artigos cientificos, elaborados entre os anos de 2012 a 2021. Além disso, a pesquisa pode ser caracterizada como de cunho qualitativo em que são analisados subjetivamente os resultados de outras produções científicas acerca da temática. Conforme a pesquisa idetificou-se a Comunicação Alternativa Ampliada pode ser considerada uma intervenção facilitadora na interação social e na qualidade de vida de pacientes com Esclerose Lateral Amiotrófica.
\end{abstract}

Palavras-chave: Comunicação; ELA; Fonoaudiologia.

\begin{abstract}
Alternative Communication is a communication system that provides individuals, at different stages of life, with the possibility of communicating in different social contexts. Thus, several resources are used to contribute to the individual with Amyotrophic Lateral Sclerosis - ALS, which is a disease characterized by deterioration of motor neurons and causes progressive paralysis of skeletal muscles, compromising limb motricity, swallowing, breathing and speech. Speech impairment plays a fundamental role in the patient's quality of life as well as that of their caregiver. The Amplified Alternative Communication (AAC) appears as an opportunity to alleviate the communication limitations imposed by the disease, due to the difficulties presented by the disease, means that facilitate the continuity of daily activities are necessary. The present work aims to analyze alternatives, for the expression of thought, to a person who is prevented, due to the disease, from establishing effective oral, written or signed verbal communication. The research methodology consists of an integrative bibliographic research, through the analysis of theories, present in various sources, such as scientific articles, prepared between the years 2012 to 2021.
\end{abstract}


Furthermore, the research can be characterized as of qualitative nature in which the results of other scientific productions on the subject are subjectively analyzed. As the research identified, the Extended Alternative Communication can be considered a facilitating intervention in social interaction and in the quality of life of patients with Amyotrophic Lateral Sclerosis.

Keywords: Communication; ELA; Speech therapy.

\section{Resumen}

La Comunicación Alternativa es un sistema de comunicación que brinda a las personas, en diferentes etapas de la vida, la posibilidad de comunicarse en diferentes contextos sociales. Así, se utilizan varios recursos para contribuir al individuo con Esclerosis Lateral Amiotrófica - ELA, que es una enfermedad caracterizada por el deterioro de las neuronas motoras y causa una parálisis progresiva de los músculos esqueléticos, comprometiendo la motricidad de las extremidades, la deglución, la respiración y el habla. La alteración del habla juega un papel fundamental en la calidad de vida del paciente así como en la de su cuidador. La Comunicación Alternativa Amplificada (CAA) aparece como una oportunidad para paliar las limitaciones comunicativas que impone la enfermedad, debido a las dificultades que presenta la enfermedad, son necesarios medios que faciliten la continuidad de las actividades diarias. El presente trabajo tiene como objetivo analizar alternativas, para la expresión del pensamiento, a una persona a la que, debido a la enfermedad, se le impide establecer una comunicación verbal efectiva, oral, escrita o por señas. La metodología de investigación consiste en una investigación bibliográfica integradora, a través del análisis de teorías, presentes en diversas fuentes, como artículos científicos, elaborados entre los años 2012 a 2021. Además, la investigación se puede caracterizar como de carácter cualitativo en la que los resultados de se analizan subjetivamente otras producciones científicas sobre el tema. Como identificó la investigación, la Comunicación Alternativa Extendida puede considerarse una intervención facilitadora en la interacción social y en la calidad de vida de los pacientes con Esclerosis Lateral Amiotrófica.

Palabras clave: Comunicación; ELA; Terapia del lenguaje.

\section{Introdução}

A Esclerose Lateral Amiotrófica (ELA) é uma doença neurodegenerativa progressiva, idiopática e fatal que afeta os neurônios motores na medula espinal, troncocerebral e córtex motor. Trata-se de uma patologia de difícil diagnóstico precoce e terapêutica dependente de uma atenção multidisciplinar, tendo prognóstico positivo em relação aos tratamentos paliativos (Coimbra, et al., 2018).

Um conjunto de variáveis tem sido investigado como possíveis fatores de risco para ELA, considerando desde causas genéticas, 5 a 10\% com padrão de herança autossômica dominante, até fatores ambientais, químicos e físicos. Entretanto cerca de $90 \%$ são considerados esporádicos e com etiologia desconhecida (Silva, 2012).

A Comunicação Alternativa (CA), segundo a Américan Speech - Languagem - Hering Association (ASHA), destinase a compensar e facilitar permanentemente ou não, prejuízos e incapacidades dos sujeitos com graves distúrbios da compreensão e da comunicação expressiva (gestual, falada e/ou escrita). Sendo a CA uma área clínica, educacional e de pesquisa.

Além disso, é um sistema de comunicação que proporciona ao indivíduo, em diferentes fases da vida, a possibilidade de se comunicar em diversos contextos sociais. Dentro da abordagem da comunicação, vários recursos são utilizados para auxiliar com o indivíduo com Esclerose Lateral Amiotrófica - ELA, devido às dificuldades apresentadas pela doença é necessário algumas maneiras de facilitar as atividades cotidianas do indivíduo, visando proporcionar uma melhor qualidade de vida e adaptar o ambiente à sua condição (Light \& Mcnaughton, 2014).

Assim, o objetivo do referente trabalho é analisar a eficácia da comunicação alternativa em indivíduos com esclerose lateral amiotrófica. Para tanto, faz-se necessário responder ao seguinte questionamento: “Quais as contribuições do uso da comunicação alternativa em indivíduos com esclerose lateral amiotrófica? 


\section{Referencial teórico}

\subsection{A Esclerose Lateral Amiotrófica (ELA)}

Conceituada por Dorland (2006), a Esclerose Lateral Amiotrófica (ELA) "é considerada uma neuropatia genética caracterizada pela destruição progressiva dos neurônios motores que controlam a atividade dos músculos envolvidos nos movimentos voluntários e involuntários (atividade reflexa)". Dessa forma, essa doença tem como fator principal a diminuição de forma gradativa da força muscular, causando sintomas como: atrofiamento dos músculos, transtornos fonológicos e deglutição, paralisia e crises respiratórias, entre outros sintomas.

De acordo com Leite Neto, et al., (2021), incidência de ELA na população mundial está na faixa de 1,5 e 2,5 casos por ano a cada 100.000 habitantes. É mais comum no sexo masculino, após os 40 anos a sobrevida está em torno de 3 a 5 anos tendo como principal causa da morte, a insuficiência respiratória e em alguns casos apresentam sobrevida de mais de 10 anos. A estimativa é que no Brasil cerca de quinze mil pessoas possuem diagnóstico de ELA (Leite neto, et al., 2021).

Por seguinte, para Leite Neto, et al., (2017) a Esclerose Lateral Amiotrófica conhecida como ELA, pode ser diagnosticada quando o paciente apresenta dificuldade na comunicação, podendo ser notada desde os estágios iniciais da doença onde o paciente pode apresentar o sintoma de rouquidão e de acordo com o avanço da doença pode apresentar impedimento vocálico até a perda total da habilidade de comunicação e forma verbal.

Diante disso, compreende-se que a Esclerose Lateral Amiotrófica (ELA), é uma doença identificada por apresentar dificuldades na fala, à vista disso, logo ao ser diagnosticada nas primeiras fases, é necessária uma intervenção de forma imediata com o intuito amenizar o quadro evolutivo da doença e adequar os tratamentos para suprir a necessidade conforme a demanda de cada paciente.

Para Silva, (2012, p. 100) "os déficits, na maioria das vezes, são estritamente motores, não envolvendo a capacidade mental e psíquica do afetado". Contudo, a sobrevivência média varia entre 2 e 4 anos, após o início dos sintomas, aproximadamente $50 \%$ dos pacientes morrem dentro de 30 meses, somente 5 a $10 \%$ sobrevivem por 10 anos e os déficits progressivos podem levar a perda da independência (Chió, et al., 2013).

De acordo com Coimbra, et. al., (2018, p. 23) "pessoas com ELA, como qualquer outra pessoa, precisam se comunicar para interagir socialmente com familiares, amigos e cuidadores, para reduzir a ansiedade e para cuidar das suas necessidades básicas". Em conformidade a isso, as intervenções acontecem com o objetivo de obter resultados positivos, na busca de alternativas para haver uma comunicação receptiva e expressiva.

Diante das várias dificuldades proporcionadas pela doença é necessário criar meios que facilitem a continuidade das atividades cotidianas, proporcionando a esses indivíduos uma melhor qualidade de vida e maiores oportunidades da adaptação do ambiente à sua condição física (Coimbra, et. al., 2018, p. 23).

A partir disso, percebe-se que como meio primordial para interagir com outras pessoas, a comunicação deve ser realizada de várias formas, desde a fala, escrita, símbolos e gestos, mais desde que seja compreendida com o seu interlocutor dentro de um contexto entre ambos.

Portanto, oportunizar o indivíduo a adquirir um meio para se comunicar, seja da forma mais simples e de acordo com suas necessidades básicas, proporciona uma melhor qualidade de vida. Com isso, Moraes, et. al., (2019, p. 3) ressalta que "privar condições alternativas de comunicação para alguém com perda de habilidades comunicativas seria como aprisioná-lo no isolamento social e emocional, sem chances de desenvolver sua potencialidade".

Diante do exposto, fica compreendido que é essencial a comunicação, desde a forma mais simples à mais complexa, a comunicação se faz presente em todos os momentos da vida do ser humano se fazendo condição básica para a qualidade de vida. 


\subsection{A comunicação alternativa como suporte aos indivíduos com Esclerose Lateral Amiotrófica}

A comunicação alternativa (CA) é uma área de conhecimento que surgiu na década de 70, com o objetivo de auxiliar pessoas que apresentavam dificuldades e incapacidades para se comunicar. De acordo com Reily (2004, p. 88) "Esse tipo de comunicação envolve expressões faciais, corporais, gestos indicativos e representativos, voz digital, símbolos gráficos como figuras, fotos, além do uso de objetos concretos".

Dessa forma, a CA está pautada nas necessidades e capacidades desses indivíduos, por meio de simbologias, grafias, frases pré-formadas, dentre outros métodos de comunicação que não ocontecem de forma tradicional (fala e gestos). Nesse sentido, Coimbra, et al., (2018) releva que a comunicação pode ser executada por meio da tecnologia de baixo e alto custo, com o propósito de promover independência, interação social, autoestima, e claro, melhor qualidade de vida.

Outrossim, a Comunicação Alternativa se mostra um bom exemplo de tecnologia assistiva, no que se refere ao auxílio na interação e participação social desses indivíduos, promovendo oportunidades de ampliação e/ou substituição da linguagem verbal e não verbal dos indivíduos que apresentam limitações na fala, nos gestos, nas expressões faciais e na escrita. Em relação a Comunicação Alternativa Ampliada (CAA):

Entende-se que sua efetividade acontecerá por meio de uma avaliação extremamente específica do indivíduo, identificando as mais diversas limitações e potencialidades perante seu contexto e história de vida, além da aceitação e adaptação dos indivíduos ao uso da CAA (Coimbra, et. al., 2018, p. 23).

Dessa forma, Nunes (2003) ressalta que quando o indivíduo demonstra incapacidade no desenvolvimento da fala ou a fala promovida por ele não é capaz de concluir a comunicação de forma compreensiva, torna-se necessário o uso de recursos alternativos a fim de promover a interação por comunicação do indivíduo com o ambiente que ele habita. Entrelaçado a isso, Moreschi e Almeida (2012, p. 662) afirmam que: "sistemas de comunicação tornam-se alternativas na promoção de uma comunicação mais efetiva entre indivíduo sem comunicação oral e seu par, proporcionando meios funcionais de construção e partilha de ideias e sentimentos".

Segundo Tetzchner e Martinsen (2000), conforme a necessidade de cada pessoa, torna-se possível identificar quais os recursos de comunicação alternativos podem ser utilizados, além disso, para melhor identificar o nível de necessidade de cada sujeito. Para melhor evidenciar, os autores dividiram as pessoas em três grupos conforme suas necessidades, sendo assim, o primeiro grupo é constituído por pessoas que precisam de um meio para expressar-se, geralmente, demonstram diferentes capacidades de compreensão e na produção da falar, o segundo grupo são das pessoas que necessitam de um apoio de linguagem e esse grupo foi dividido de acordo com duas situações, a primeira são de pessoas que já utilizam a comunicação alternativa e ampliada para melhor aquisição da linguagem e a segunda são pessoas que conseguem verbalizar mas enfrentam dificuldades para serem compreendidas e o terceiro grupo são de pessoas que só conseguem desenvolver a linguagem falada com o uso de recursos de comunicação alternativa, pois não possuem a convencionalidade da fala para realizar a comunicação.

Diante disso, a comunicação alternativa é entendida como uma necessidade, sendo um direito legal e básico para viver. Por meio dela é possível se expressar, compartilhar ideias, estabelecer relações sociais, desenvolver habilidades e competências. À vista disso, segundo Moraes, et. al., (2019, p. 2) "indivíduos que enfrentam a barreira da comunicação apresentam menores chances de desenvolverem satisfatoriamente muitos campos da vida".

Desse modo, as contribuições teóricas dos autores supracitados, deixa claro a necessidade de Comunicação Alternativa para indivíduos que não conseguem se comunicar de maneira convencional, em específico, pacientes com Esclerose Lateral Amiotrófica. Além de ser imprescindível, a comunicação é também um direito de todo indivíduo, que precisa desta para se desenvolver em diversos âmbitos da vida. 


\section{Metodologia da pesquisa}

O percurso metodológico desta pesquisa foi realizada alicerçada em uma revisão integrativa, a qual permite o conhecimento atual da temática, por ser constituída através da análise, identificação e sintetização dos resultados de estudos realizados (Souza, et al., 2010).

Dito isso, foi realizada uma pesquisa na base de dados: LILACS (Literatura Latina Americana); SCIELO (Scientífic Eletrônic Library Online), Medline (Medical Literature analysis and Retrievel System Online), e Capes, em busca de estudos que apresentassem temas relacionados à pesquisa em evidência. Como critério de inclusão dos artigos científicos,foram selecionados os textos completos e disponíveis gratuitamente, nos idiomas português, inglês e espanhol, dentro do recorte temporal dos anos de 2012 a 2021. A pesquisa foi realizada entre os meses de outubro e novembro do ano de 2021.

Para a realização da busca das fontes para fundamentar a pesquisa foram utilizados os seguintes descritores em saúde: “esclerose lateral amiotrófica" "sistemas de comunicação alternativos e aumentativos","fonoaudiologia", sendo combinados com o operador booleano AND, "esclerose lateral amiotrófica AND sistemas de comunicação alternativos e aumentativos", "Esclerose Lateral Amiotrófica AND fonoaudiologia", "sistemas de comunicação alternativos e aumentativos AND fonoaudiologia". E como critério de exclusão foram selecionados os artigos que apresentassem apenas resumos, outros tipos de produção, como: notícias, blogs e sites, trabalhos que fugissem à temática apresentada, e fora do recorte temporal. Os artigos que estão disponíveis em mais de uma base de dados foram contabilizados apenas uma vez.

Para uma maior compreensão sobre o critério de seleção dos artigos utilizados na presente pesquisa houve a necessidade da elaboração do seguinte fluxograma (Figura 1), o qual demonstra as bases de dados (SCIELO, CAPES e MEDLINE), o número de artigos encontrados e o quantitativo de artigos foram excluídos.

Figura 1: Quantitativo de artigos utilizados na pesquisa.

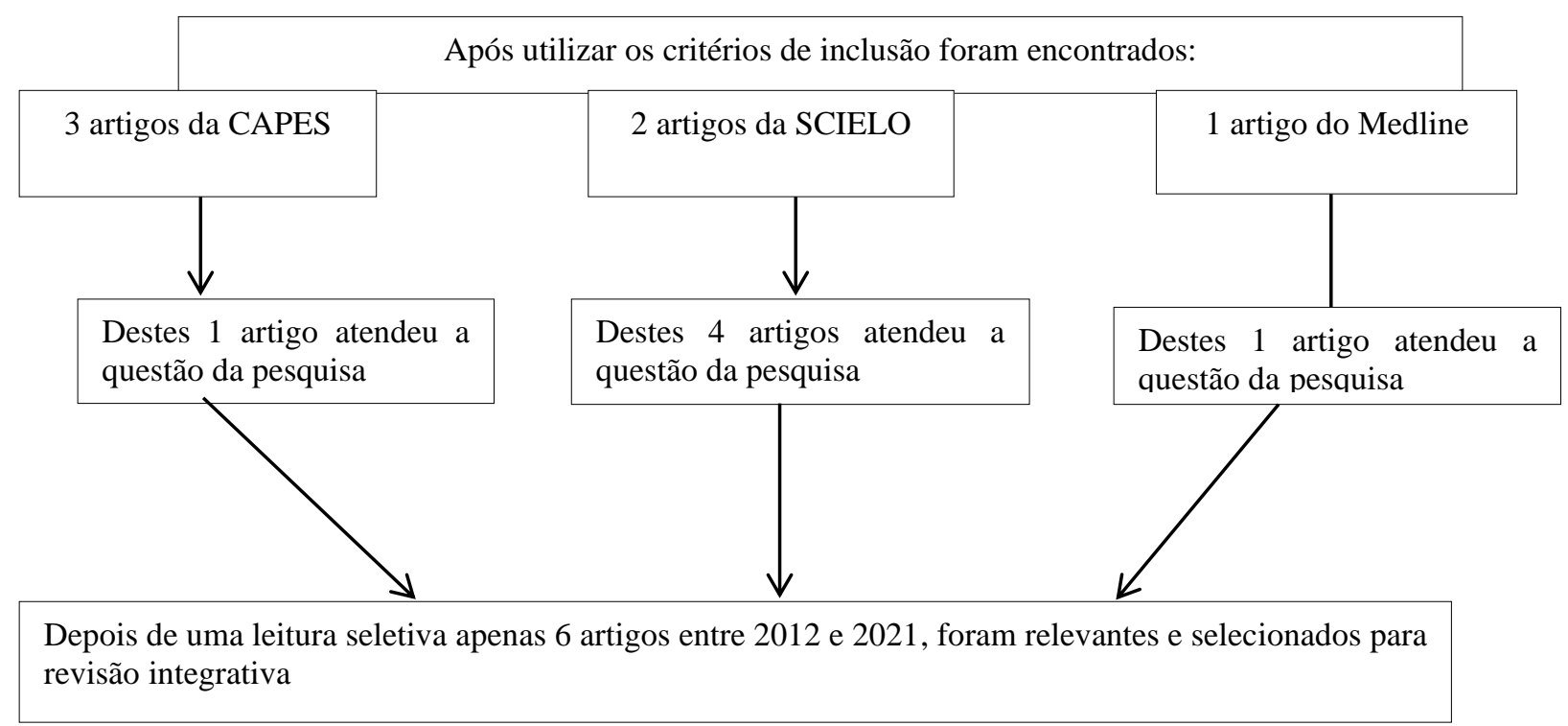

Fonte: Autores (2021).

Posteriormente a seleção dos artigos utilizados na presente pesquisa houve a necessidade da elaboração de um quadro identificando os seus autores, a base de dados na qual está disponibilizado o artigo, a metodologia e os resultados presentes nas fontes consultadas (Quadro 1). 


\section{Resultados e Discussão}

A compilação sintética dos seis artigos selecionados no (Quadro 1) demonstra de modo objetivo, conforme evidenciou na pesquisa as principais contribuições da Comunicação Alternativa.

Quadro 1. Artigos sobre os Benefícios do uso da Comunicação Alternativa em indivíduos com esclerose lateral amiotrófica.

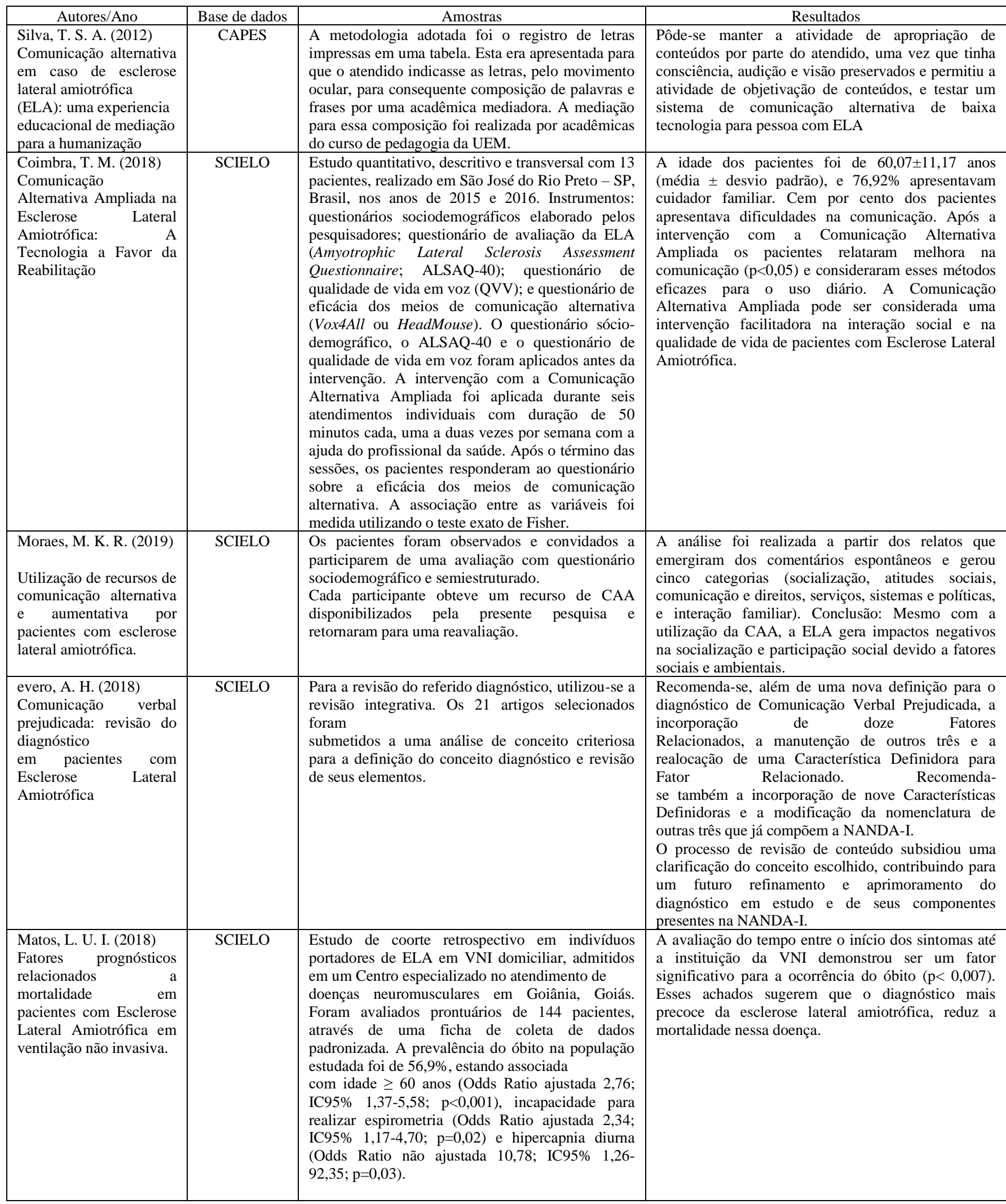




\begin{tabular}{|c|c|c|c|}
\hline $\begin{array}{l}\text { Oliveira, A. M. F. } \\
\text { (2019) } \\
\text { Sistemas Aumentativos e } \\
\text { Alternativos } \\
\text { de Comunicação na } \\
\text { Esclerose Lateral } \\
\text { Amiotrófica: } \\
\text { Aplicabilidade } \\
\text { Utilidade nos Doentes, } \\
\text { Cuidadores } \\
\text { Profissionais de Saúde }\end{array}$ & MEDLINE & $\begin{array}{l}\text { Estudo de tipo exploratório, observacional e } \\
\text { transversal cuja amostra inclui } 40 \text { doentes, } 38 \\
\text { cuidadores e } 19 \text { profissionais de saúde na área da } \\
\text { Esclerose Lateral Amiotrófica. Para a recolha de } \\
\text { dados foi utilizada a escala de avaliação funcional } \\
\text { ALSFRS-R aplicada aos doentes, bem como três } \\
\text { inquéritos distintos para os doentes, cuidadores e } \\
\text { profissionais de saúde. A investigação decorreu na } \\
\text { Associação Portuguesa de Esclerose Lateral } \\
\text { Amiotrófica e no Hospital de Santo António - } \\
\text { Centro Hospitalar e Universitário do Porto. Os } \\
\text { procedimentos formais e éticos foram respeitados, } \\
\text { obtendo a autorização das instituições e o } \\
\text { consentimento informado dos participantes para a } \\
\text { realização do estudo. }\end{array}$ & $\begin{array}{l}\text { Os resultados mostraram que a percepção da utilidade } \\
\text { dos Sistemas Aumentativos e Alternativos de } \\
\text { Comunicação é elevada. Constatou-se a existência de } \\
\text { fatores preponderantes para uma maior utilidade dos } \\
\text { sistemas. Porém, a literacia acerca do tema é escassa, } \\
\text { demonstrando a necessidade de uma maior } \\
\text { conscientização para esta temática. }\end{array}$ \\
\hline
\end{tabular}

Fonte: Autores (2021).

Após análise minuciosa das teorias presentes, principalmente em artigos científicos, consultas em sítios eletrônicos, dentre outras fontes, referentes à comunicação alternativa como suporte a indivíduos com esclerose lateral amiotrófica chegouse a uma serie de resultados.

De uma forma geral por meio de comunicação alternativa como suporte a indivíduos com esclerose lateral amiotrófica é possível amenizar os problemas oriundos dessa enfermidade, como o enfraquecimento dos músculos responsáveis pela respiração gera incapacidade respiratória progressiva, causando fadiga e falta de ar a pequenos esforços, principalmente em posição deitada. A fadiga dificulta a fala, que por sua vez passa a ser cada vez mais cansativa, pausada e incompreensível. Outro fator contribuinte para a aceleração da incompreensão da fala é o comprometimento da musculatura da face, já que a função da fala depende do bom e coordenado desempenho motor da língua, dos lábios, e dos maxilomandibulares (Coimbra, et al., 2018).

A comunicação alternativa também é importante na questão de intervir em um dos piores comprometimentos causados pela ELA, e que este interfere na participação social que passa a ser cada vez mais restrita, causa fragilidade nas decisões, baixa qualidade de vida e desenvolvimento de problemas emocionais $(8,12)$, sendo possível observar que com o aumento da dependência, alguns pacientes passam a demonstrar menos suas vontades e desejos, não só pela dificuldade comunicativa, mas por medo de gerar sobrecarga familiar (Meily, 2017).

Além disso, a comunicação alternativa é importante devido à condição de restrição ao domicílio. Ocorrendo dentre os principais motivos de restrição desses pacientes as dificuldades que surgem de acordo à progressão da doença, como a dificuldade respiratória e as limitações físicas, a dificuldade de deslocamento ou locomoção, bem como por fragilidade emocional (Silva, 2012).

Conforme a pesquisa observa-se que são vários os motivos que levam a restrição na participação da pessoa com ELA e que além, dos que estão associados com a evolução da enfermidade, há também outros fatores envolvidos, como educação, exigências comunicativas nos ambientes de convívio, fator emocional, cognição. Entretanto, determina que o nível de comprometimento da fala seja um dos fatores mais determinante neste processo (Matos, 2018).

Pessoas com esclerose apresentam alto nível de dificuldade para se comunicar sem o recurso de comunicação e alto nível de importância na melhora do desempenho comunicativo. Mesmo com a piora do quadro clínico os pacientes apresentam redução da dificuldade comunicativa ao utilizar a CAA, apresentando assim benefícios comunicativos em relação ao seu uso (Oliveira, 2019).

Em alguns casos, entretanto não é possível o uso efetivo do dispositivo de comunicação sem as adequações posturais providenciadas, pois somente dessa forma poderá haver uma maior aceitação e utilização do recurso, além de uma melhora na comunicação com familiares, amigos e profissionais de saúde. A perda das habilidades comunicativas gera fragilidade emocional e impactos negativos na socialização e participação social (Leite Neto, et al., 2021). 
Os pacientes apresentam boa aceitação do novo recurso de CAA, apresentando comunicação eficaz principalmente em ambiente familiar. Um fator contribuinte se apresenta no empenho para priorizar a necessidade e vontade individual de cada paciente em todas as fases do processo de prescrição a utilização da CAA, além do treinamento, suporte e acompanhamento fornecido enquanto o paciente necessitar. Embora necessitem de adaptação postural para utilizar o recurso, sendo imprescindíveis as adaptações desenvolvidas (Parreira, et al., 2019).

Entretanto, Ribeiro (2007) revela que a ELA gera impactos negativos na socialização e participação social devido não só à dificuldade em administrar uma comunicação causada pelas limitações advindas da doença, mas também em virtude da incapacidade do ciclo social do paciente em particular para desenvolver interação e comunicação eficaz com a pessoa que apresenta essa condição, e da incapacidade de alguns pacientes no enfrentamento emocional das barreiras encontradas em seus ciclos sociais. A fragilidade emocional pode ter relação com a própria condição de saúde, com as barreiras sociais, familiares e ambientais, como a dificuldade em obter seus direitos para uma vida digna e de qualidade (Moraes, 2019).

As principais dificuldades no acesso a esses recursos incluíram a baixa condição financeira e o baixo nível de informação em relação à CAA. É fundamental que gestores e profissionais de saúde, dentro de sua abordagem profissional, estejam familiarizados e capacitados acerca da CAA, para oferecerem suporte e informação para pessoas que apresentam perda de habilidades comunicativas. Também é de suma importância que o paciente participe de todo o processo de indicação desses dispositivos de comunicação e posteriormente receba o treinamento para a utilização e adequada assistência e acompanhamento do uso dessas tecnologias (Carvalho, 2015).

Os procedimentos de comunicação alternativa é uma situação inovadora e propor, no campo da educação, da pedagogia, um trabalho em prol da continuidade do processo de humanização da pessoa em situação de degeneração biológica extrema é impactante, seja pelos resultados já em curso, seja por outros desdobramentos, de natureza didática acadêmica (Silva, 2012).

Em pacientes com ELA, a intervenção com a CAA melhora significativamente a comunicação existente entre o paciente e o seu respectivo cuidador no contexto terapêutico. No entanto, mesmo com a modernização e uma maior disponibilidade para o tratamento médico e para a reabilitação, ainda considera- se uma realidade muito longe do perfil socioeconômico da sociedade vigente. Há a necessidade de que os profissionais da saúde estejam continuamente atualizados e informados no que se refere aos direitos dessa população para que obstáculos criados socialmente possa ser apaziguada e as pessoas com ELA tenham a acessibilidade de maneira efetiva e, por conseguinte, melhorar a qualidade de vida das pessoas (Coimbra, et al., 2018).

É grande o prejuízo na produção da fala, da voz ou da escrita em pessoas com ELA devido à dificuldade em transmitir e/ou compreender uma mensagem. Recomenda-se a realocação de uma característica definidora para fator relacionado, a incorporação de fatores relacionados e a manutenção de outros.

Assim, dentre os fatores relacionados, ressaltam-se: a degeneração de neurônios motores; traqueostomia; labilidade emocional; dispneia; paresia muscular espástica facial; paresia muscular flácida facial; paresia da língua; velocidade da língua reduzida; hiperadução de pregas vocais; hipoadução de pregas vocais; fraqueza da musculatura respiratória; incompetência velofaríngea; atrofia muscular da língua; fasciculações na língua; sialorreia e déficits cognitivos. É fundamental, ainda, a realização da validação por especialistas e da validação clínica para a confirmação dos resultados encontrados envolvendo pacientes com ELA (Severo, 2018).

Ainda hoje existem dificuldades em relação a um número pequeno de pessoas que usam a comunicação alternativa, porém o presente estudo pode servir como uma fonte em prol de corroborar com estudos sobre a prática clínica e o aperfeiçoamento do uso de recursos por pessoas com ELA ou outras condições de saúde que comprometam a comunicação. 
Sugere-se novas pesquisas que investiguem fatores relacionados a aplicação prática e sucesso de utilização dos recursos de comunicação em um número maior de pacientes com ELA.

\section{Considerações Finais}

Conforme a presente pesquisa evidenciou-se que em pacientes com ELA, a intervenção com a CAA melhora significativamente a comunicação entre paciente e os seus respectivos cuidadores no contexto terapêutico e social. No entanto, mesmo com a alta modernização e uma disponibilidade mais ampla para o tratamento médico e de reabilitação, ainda hoje é uma realidade extremamente distante diante do perfil socioeconômico da sociedade brasileira.

A Comunicação Alternativa e Ampliada (CAA) é uma categoria de Tecnologia Assistiva (TA) que busca proporcionar a comunicação eficaz e a participação social através de ferramentas, de métodos, de expressão facial, de símbolos, de programas de computador simples ao mais avançado, sendo direcionada para a solução ou ampliação de qualquer perda de habilidade comunicativa como dificuldade de fala ou distúrbios de linguagem, e déficit de desenvolvimento e intelectual.

Sendo assim, há necessidade de que os profissionais da saúde, como os fonoaudiólogos, estejam continuamente buscando atualização e informação no que se refere aos direitos dessa população, pois somente dessa forma a barreira criada socialmente possa ser apaziguada e as pessoas com ELA possuam a total acessibilidade efetivamente e, por conseguinte, obter uma melhor qualidade de vida. Além do mais, sugere-se a continuidade de estudos futuros sobre os benefícios do uso da Comunicação Alternativa aos indivíduos com ELA.

\section{Referências}

Carvalho, M. G. R. (2015). Dispositivos de tecnologia assistiva: fatores relacionados ao abandono. Cadernos de Terapia Ocupacional da UFSCar. 2015 ; 23 (3): 611-624.

Chiò, A., Logroscino, G., Traynor, B. J., Collins, J., Simeone, J. C., \& Goldstein, L. A. (2-13). Global epidemiology of amyotrophic lateral sclerosis: a systematic review of the published literature. Neuroepidemiology. 2013;41(2):118-30. DOI: 10.1159/000351153.

Coimbra, T., Ezequiel, C., Moreira, D., Morita, M., Castiglioni, L., \& Bianchin, M. (2018). Comunicação Alternativa Ampliada na Esclerose Lateral Amiotrófica: A Tecnologia a Favor da Reabilitação. Arquivos De Ciências Da Saúde, 25(3), 22-26. doi:10.17696/2318-3691.

Dorland. (2006). Dicionário médico. São Paulo: Roca, 2006.

Light, J., Mcnaughton, D. (2014). Communicative competence for individuals who require augmentative and alternative communication: a new definition for a new era of communication? Augmentative and Alternative Communication. 2014; 30(1):1-18.

Leite Neto, L., Constantini, A. C., \& Chun, R. Y. S. (2017). Communication vulnerable in patients with Amyotrophic Lateral Sclerosis: a systematic review. Neuro Rehabilitation. 2017; 40 (4) 561-568.

Leite Neto, L., França Júnior, M. C., \& Chun, R. Y. S. (2021). Inteligibilidade de fala em pessoas com Esclerose Lateral Amiotrófica (ELA). CoDAS 33(1), e20190214. https://doi.org/10.1590/2317-1782/20202019214.

Matos, L. U. I. (2018). Fatores prognósticos relacionados à mortalidade em pacientes com Esclerose Lateral Amiotrófica em ventilação não invasiva. Universidade Federal de Goiás. Programa de Pós-Graduação em Ciências da Saúde. Goiânia - GO.

Meily, J. M. A. (2017). Participation restrictions in ambulatory ALS patients: physical and psychological factors. Muscle e Nerve. 56(5): 912-918.

Moraes, K. R. M. (2019). Utilização de recursos de comunicação alternativa e aumentativa por pacientes com esclerose lateral amiotrófica. Manual Therapy, Posturology \& Rehabilitation Journal.

Moresch, C. L., \& Almeida, M. A. (2012). A Comunicação Alternativa Como Procedimento de Desenvolvimento de Habilidades Comunicativas. Rev. Bras. Ed. Esp., 18(4), 661-676, https://www.scielo.br/j/rbee/a/N3Sz9N6gvhGmvZnNYPTQdwx/?lang=pt.

Nunes, D. R. \& Nunes, L. R. (2003). Efeitos dos procedimentos naturalísticos no processo de aquisição de linguagem através de sistema pictográfico em criança autista. Em L. R. Nunes (Org.), Favorecendo o desenvolvimento da comunicação em crianças e jovens com necessidades educacionais especiais (PP. 125-141). Dunya.

Oliveira, A. M. F. (2019). Sistemas Aumentativos e Alternativos de Comunicação na Esclerose Lateral Amiotrófica: Aplicabilidade e Utilidade nos Doentes, Cuidadores e Profissionais de Saúde. $2^{\circ}$ Ciclo de Estudos Mestrado em Cuidados Paliativo. Uporto. 
Research, Society and Development, v. 10, n. 15, e554101523243, 2021

(CC BY 4.0) | ISSN 2525-3409 | DOI: http://dx.doi.org/10.33448/rsd-v10i15.23243

Parreira, S. L. S., Moraes, M. K. R., Santos, V. R., Rosseto, L. P., Vento, D. A., Valle, E. F. V., \& Oliveira, A. S. B. (2019). Evaluation of alternative and augmentative communication in patients with amyotrophic lateral sclerosis, based on ICF. Manual Therapy, Posturology \& Rehabilitation Journal, 1-13. https://doi.org/10.17784/mtprehabjournal.2019.17.721.

Reily, L. (2004). Sistema de comunicação Suplementar e alternativa. In: Reily, L. Escola inclusiva: linguagem e mediação. ed. Campinas: Papirus. (série Educação Especial). Cap. 3.67 p.

Ribeiro, C. G. (2007). Esclerose lateral Amiotrófica: um impacto subjetivo na família. Brasília: Faculdade de Ciências da Saúde - FACS.

Severo, A. H. (2018). Impaired Verbal Communication: diagnosis review in patients with Amyotrophic Lateral Sclerosis. Rev Bras Enferm. 71(6):3063-73. http://dx.doi.org/10.1590/0034-7167-2017-0763.

Silva, T. S. A. (2012). Direito de acesso aos recursos de tecnologia assistiva: legislação e conhecimento de usuário. Acta Scientiarum. Education, 34(1), 99100 ,

Souza, M. T., Silva, M. D., \& Carvalho, R. (2010) . Revisão integrativa: o que é e como fazer. Einstein: 8(1), 102 - 106. https://doi.org/10.1590/S1679$45082010 \mathrm{RW} 1134$.

Tetzchner, S. V., \& Martinsen, H. (2000). Introdução à comunicação aumentativa e alternativa. Coleção Educação Especial. Editora Porto, 10. 